\title{
Pseudomonas aeruginosa uses type III secretion system to kill biofilm-associated amoebae
}

\author{
Carsten Matz ${ }^{1,2,3}$, Ana Maria Moreno ${ }^{1}$, Morten Alhede ${ }^{2}$, Mike Manefield ${ }^{1}$, Alan R Hauser ${ }^{4,5}$, \\ Michael Givskov ${ }^{2}$ and Staffan Kjelleberg ${ }^{1}$ \\ ${ }^{1}$ School of Biotechnology and Biomolecular Sciences and Centre for Marine Bio-Innovation, University \\ of New South Wales, Sydney, Australia; ${ }^{2}$ Bioscience and Technology, BioCentrum, Technical University \\ of Denmark, Lyngby, Denmark; ${ }^{3}$ Division of Cell and Immune Biology, Helmholtz Centre for Infection \\ Research, Braunschweig, Germany; ${ }^{4}$ Department of Microbiology/Immunology, Northwestern University, \\ Chicago, IL, USA and ${ }^{5}$ Department of Medicine, Northwestern University, Chicago, IL, USA
}

\begin{abstract}
Bacteria and protozoa coexist in a wide range of biofilm communities of natural, technical and medical importance. Generally, this interaction is characterized by the extensive grazing activity of protozoa on bacterial prey populations. We hypothesized that the close spatial coexistence in biofilms should allow opportunistic pathogenic bacteria to utilize their eukaryote-targeting arsenal to attack and exploit protozoan host cells. Studying cocultures of the environmental pathogen Pseudomonas aeruginosa and the amoeba Acanthamoeba castellanii, we found that $\boldsymbol{P}$. aeruginosa rapidly colonized and killed biofilm-associated amoebae by a quorum-sensing independent mechanism. Analysis of the amoeba-induced transcriptome indicated the involvement of the $P$. aeruginosa type III secretion system (T3SS) in this interaction. A comparison of mutants with specific defects in the T3SS demonstrated the use of the secretion apparatus and the effectors ExoU, ExoS and ExoT in the killing process, of which ExoU had the greatest impact. T3SS-mediated virulence towards $A$. castellanii was found to be controlled by the global regulators RpoN and RpoS and through modulation of CAMP and alginate biosynthesis. Our findings suggest that conserved virulence pathways and specifically the T3SS play a central role in bacteria-protozoa interactions in biofilms and may be instrumental for the environmental persistence and evolution of opportunistic bacterial pathogens.
\end{abstract}

The ISME Journal (2008) 2, 843-852; doi:10.1038/ismej.2008.47; published online 15 May 2008

Subject Category: microbe-microbe and microbe-host interactions

Keywords: Acanthamoeba; bacteria-protozoa interactions; conserved virulence; transcriptome

\section{Introduction}

Microbial biofilms constitute the major proportion of bacterial biomass and activity in many natural and man-made systems. At the same time, biofilms serve as important environmental reservoirs for pathogenic bacteria (Flanders and Yildiz, 2004) and are the causative agents for many persistent bacterial infections (Costerton et al., 1999). Synergistic and antagonistic interactions among microorganisms are predicted to be central to the structure, composition and function of biofilm communities (Hassell et al., 1994; Kerr et al., 2002; Battin et al., 2003). Despite the consensus that

Correspondence: C Matz, Division of Cell and Immune Biology, Helmholtz Centre for Infection Research, Inhoffenstr. 7, Braunschweig D-38124, Germany.

E-mail: carsten.matz@helmholtz-hzi.de

Received 25 February 2008; revised 24 April 2008; accepted 24

April 2008; published online 15 May 2008 biofilms represent mixed microbial communities, studies to date have addressed the physiology and regulation of biofilm bacteria almost to the exclusion of the other major players.

One group of microorganisms that lives in close association with bacterial biofilms are phagotrophic protists, the protozoa. Predation by protozoa is considered to be the major source of mortality for bacterial populations in many ecosystems (Sherr and Sherr, 2002). Protozoa, free-living amoeba in particular, are associated with bacterial biofilms in a wide range of habitats, including rivers, activated sludge, water pipes and filters, as well as dental unit waterlines and the oral cavity (Arndt et al., 2003; Marciano-Cabral and Cabral, 2003; Parry, 2004). The close association of bacteria and protozoa in biofilms and their long co-evolutionary history are thought to give rise to a series of bacterial adaptations ensuring survival and coexistence (Matz and Kjelleberg, 2005). Several bacteria, among them 
many facultative intracellular pathogens, have developed mechanisms to survive and replicate inside free-living amoebae, such as Legionella pneumophila, Francisella tularensis, many Mycobacterium spp. and Escherichia coli O157 (Molmeret et al., 2005). Besides being able to grow and survive intracellularly, bacteria may develop protective and exploitative mechanisms that act before the internalization by protozoa (Matz and Kjelleberg, 2005). The formation of biofilms and matrix-encased microcolonies has recently been reported to enhance the persistence of Vibrio cholerae and Pseudomonas aeruginosa in the presence of protozoa. This occurs because localized high bacterial densities allow the synchronized secretion of antiprotozoal compounds via quorum-sensing signals (Matz et al., 2004, 2005).

Although biofilms play roles in ensuring the persistence of human pathogens in the environment, little is known about the specific ways pathogens benefit from these associations. P. aeruginosa is a versatile gram-negative bacterium that is ubiquitous in soil, freshwater and marine habitats (Botzenhart and Döring, 1993; Kimata et al., 2004; Pirnay et al., 2005). At the same time, it is one of the most widespread opportunistic pathogens causing infections in a variety of hosts, including insects, plants, animals and humans (Bodey et al., 1993; Rahme et al., 1995; Tan et al., 1999; Plotnikova et al., 2000; Lau et al., 2003; Miyata et al., 2003; He et al., 2004). We have recently demonstrated that biofilms of $P$. aeruginosa are effectively defended from grazing by the amoeba Acanthamoeba sp. (Weitere et al., 2005). In the present study we investigated the molecular mechanisms by which $P$. aeruginosa responds to the presence of Acanthamoeba castellanii. We hypothesized that the coexistence of bacteria and protozoa in biofilms should induce either defensive or exploitative behaviour by the opportunistic pathogen P. aeruginosa.

\section{Material and methods}

Organisms and growth conditions

Bacterial strains and mutants used in this study are listed in Table 1 . The $P$. aeruginosa PAO1 wild type used was obtained from the Pseudomonas Genetic Stock Center (www.pseudomonas.med.ecu.edu, strain PAO0001). This PAO1 isolate has served as DNA source for the Pseudomonas Genome Project (www.pseudomonas.com) and, subsequently, as template for design of the $P$. aeruginosa GeneChip (Affymetrix Inc., Santa Clara, CA, USA). P. aeruginosa PAO1 transposon insertion mutants were obtained from the University of Washington Genome Center (Jacobs et al., 2003). Transposon type, insertion, location and orientation were confirmed for each of the mutants. The isogenic rhlR/lasR QS mutant was constructed by Rasmussen et al. (2005). To study the components of the type III secretion system (T3SS), we used $P$. aeruginosa PA99, a
Table 1 Strains and plasmids used in this study

\begin{tabular}{|c|c|c|}
\hline $\begin{array}{l}\text { Strain or } \\
\text { plasmid }\end{array}$ & $\begin{array}{l}\text { Relevant } \\
\text { characteristic }\end{array}$ & Reference \\
\hline \multicolumn{3}{|l|}{ Bacteria } \\
\hline \multicolumn{3}{|l|}{$\begin{array}{l}\text { Pseudomonas } \\
\text { aeruginosa }\end{array}$} \\
\hline \multirow[t]{2}{*}{ PAO1 } & Wild-type ATCC & American Type \\
\hline & 15962 & $\begin{array}{l}\text { Culture } \\
\text { Collection }\end{array}$ \\
\hline rhlR/lasR & $\begin{array}{l}\text { PAO1 rhlR::Tc }{ }^{\mathrm{r}} \\
\text { lasR::Gm }{ }^{\mathrm{r}}\end{array}$ & $\begin{array}{l}\text { Rasmussen } \\
\text { et al., } 2005\end{array}$ \\
\hline$v f r$ & $\mathrm{PAO} 1 \Delta v f r$ & $\begin{array}{l}\text { University of } \\
\text { Washington } \\
\text { Genome Center }\end{array}$ \\
\hline rpos & PAO1 1 rpos & $\begin{array}{l}\text { University of } \\
\text { Washington } \\
\text { Genome Center }\end{array}$ \\
\hline rpoN & PAO1 rpoN::Gm ${ }^{\mathrm{r}}$ & $\begin{array}{l}\text { Thompson } \\
\text { et al., } 2003\end{array}$ \\
\hline mucA22 & $\begin{array}{l}\text { Point mutation in } \\
\text { mucA of PAO1 }\end{array}$ & $\begin{array}{l}\text { Mathee et al., } \\
1999\end{array}$ \\
\hline PA99 & Clinical isolate & $\begin{array}{l}\text { Feltman et al., } \\
2001\end{array}$ \\
\hline PA99S & PA99 & $\begin{array}{l}\text { Shaver and } \\
\text { Hauser, } 2004\end{array}$ \\
\hline PA99T & $\begin{array}{l}\text { PA99null } \\
\text { complemented } \\
\text { with mini-CTX1- } \\
\text { ExoT }\end{array}$ & $\begin{array}{l}\text { Shaver and } \\
\text { Hauser, } 2004\end{array}$ \\
\hline PA99U & 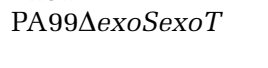 & $\begin{array}{l}\text { Shaver and } \\
\text { Hauser, } 2004\end{array}$ \\
\hline PA99secr $^{-}$ & $\mathrm{PA} 99 \Delta p s c \mathrm{~J}$ & $\begin{array}{l}\text { Shaver and } \\
\text { Hauser, } 2004\end{array}$ \\
\hline \multicolumn{3}{|l|}{ Plasmids } \\
\hline pMF230 & $\begin{array}{l}\text { pMF36 containing } \\
\text { gfp mut2; } \mathrm{Cb}^{\mathrm{r}}\end{array}$ & $\begin{array}{l}\text { Franklin and } \\
\text { Ohman, } 1993\end{array}$ \\
\hline $\begin{array}{l}\text { mini-CTX1- } \\
\text { ExoT }\end{array}$ & $\begin{array}{l}\text { exoT in mini-CTX1; } \\
\text { Tet }^{\mathrm{r}}\end{array}$ & $\begin{array}{l}\text { Shaver and } \\
\text { Hauser, } 2004\end{array}$ \\
\hline \multicolumn{3}{|l|}{ Protozoa } \\
\hline $\begin{array}{l}\text { Acantha- } \\
\text { moeba } \\
\text { castellanii }\end{array}$ & $\begin{array}{l}\text { Wild-type ATCC } \\
30234\end{array}$ & $\begin{array}{l}\text { American Type } \\
\text { Culture } \\
\text { Collection }\end{array}$ \\
\hline
\end{tabular}

well-characterized clinical isolate that naturally carries the exoU, exoS and exoT genes, but lacks exoY (Feltman et al., 2001). Bacterial strains were routinely grown on Luria-Bertani agar, and, prior to the experiments, on a $10 \%$ dilution of M9 medium (Sambrook et al., 1989) supplemented with $20 \mathrm{mM}$ glucose. Axenic cultures of A. castellanii ATCC 30234 were maintained as confluent monolayers in tissue culture flasks on M9-based peptone/yeast extract/glucose (PYG) medium at $30^{\circ} \mathrm{C}$. For the experiments, trophozoites were suspended from the flask bottom (incubation on ice for $30 \mathrm{~min}$ ), washed twice by centrifugation $(500 \mathrm{~g}, 5 \mathrm{~min})$ and quantified photometrically by $\mathrm{OD}_{600}$ measurements.

\section{Flow-cell experiments}

Flow-cell experiments were performed as described previously (Weitere et al., 2005) at a constant 
temperature of $22^{\circ} \mathrm{C}$. Flow channels were inoculated with overnight cultures of the $P$. aeruginosa PAO1 wild type and the isogenic lasR/rhlR mutant and incubated without flow for $1 \mathrm{~h}$ to allow bacteria to attach. The biofilms were grown without amoebae for 3 days in 10\% M9 medium (supplemented with $0.3 \mathrm{~mm}$ glucose). On day 4 , washed cells of $A$. castellanii were resuspended in 10\% M9 medium at a final concentration of $10^{3}$ cells per $\mathrm{ml}$ and injected into three replicate flow chambers. Amoebae numbers and biofilm structures were monitored daily by phase contrast and scanning confocal laser microscopy, respectively. In each flow chamber, amoebae were counted in 15 randomly distributed $x-y$ grids. The experiment was repeated for the $P$. aeruginosa PA99 wild type and the isogenic PA99secr ${ }^{-}$mutant under identical conditions.

Transcriptome profiling of $\mathrm{P}$. aeruginosa biofilms The transcriptional response of $P$. aeruginosa biofilms to $A$. castellanii was profiled by using PAO1 Affymetrix GeneChips (Affymetrix Inc., Santa Clara, CA, USA). Biofilms were grown at $22^{\circ} \mathrm{C}$ for 3 days in continuous-culture silicon tubing (length: $12 \mathrm{~cm}$; inner diameter: $0.8 \mathrm{~cm}$; total volume: $6 \mathrm{ml})$. Washed cells of $A$. castellanii were resuspended in $6 \mathrm{ml}$ of $10 \% \mathrm{M9}$ medium at a final concentration of $10^{6}$ cells per $\mathrm{ml}$ and injected into the flow chamber. After $2 \mathrm{~h}$ of co-incubation, the excess fluid inside the tube was discarded and the biofilm cells were harvested mechanically and collected in two volumes of RNAlater (Qiagen $\mathrm{GmbH}$, Hilden, Germany) to be stored at $-80^{\circ} \mathrm{C}$. The RNAlater-suspended biofilms were sonicated to dissolve clumped cells. Amoebae and bacteria were separated by centrifugation. RNA was extracted from the harvested bacterial cells with the RNeasy Mini Purification kit (Qiagen $\mathrm{GmbH}$ ) and contaminating chromosomal DNA was removed by RQ1 RNase-free DNase treatment. RNA was converted to cDNA (Random primers, RT SuperScript III, Invitrogen Corporation, Carlsbad, CA, USA) and fragmented. The fragments were labelled with Biotin-ddUTP (EnzoBioArray terminal labelling kit; Invitrogen Corporation) and hybridized to the GeneChip $P$. aeruginosa Genome Array (Affymetrix Inc.). Microarray data were analysed with Affymterix GeneChip Operating Software and Microsoft Excel. To identify a common set of amoeba-responsive genes, the experiment was repeated twice. Those genes that showed a statistically significant $(P<0.05)$ change in expression and a more than threefold change in magnitude when grown in the presence of $A$. castellanii relative to M9 medium alone were regarded as significant. The less stringent cut-off value was chosen because of the reported heterogeneous nature of biofilms to allow gene expression patterns of subpopulation to be recorded in the analysis.
Coculture batch experiments

Batch experiments of $A$. castellanii and $P$. aeruginosa were conducted in flat bottom, tissue culture-treated 96-well plates (Corning Incorporated). Tissue culture plates containing M9-based PYG medium were inoculated with A. castellanii and incubated at $30^{\circ} \mathrm{C}$ for 2 days. Prior to the experiment, the PYG medium was carefully removed from the wells and the cells were gently washed with 10\% M9 medium. Cultures of $P$. aeruginosa were grown on $10 \%$ M9 medium supplemented with $0.2 \%$ glucose to an $\mathrm{OD} \approx 0.1$. For the experiment, bacterial suspensions were adjusted to $\mathrm{OD}=0.025$ by diluting the culture with the filtered culture supernatant. Wells containing washed $A$. castellanii were then inoculated in the following arrangement per $P$. aeruginosa strain: three replicate wells with $75 \mu \mathrm{l}$ of the bacterial culture of OD $=0.025$ (treatment wells), three replicate wells with $75 \mu \mathrm{l}$ of $10 \%$ M9 medium without glucose (medium-only control) and three replicate wells with $75 \mu \mathrm{l}$ of bacteria-free supernatant (supernatant control). The plates were incubated at $30{ }^{\circ} \mathrm{C}$ for $24 \mathrm{~h}$. Fixing was done by adding $75 \mu \mathrm{l}$ of $4 \%$ glutaraldehyde to each well to get a final concentration of $2 \%$ glutaraldehyde. The plates were sealed with Parafilm and stored at $4{ }^{\circ} \mathrm{C}$ until they were examined using an inverted light microscope (Olympus). The number of amoeba was obtained by counting the numbers of cells in one transect per well using a magnification of $\times 30$.

\section{Statistical analysis}

Changes in amoebae numbers over time were tested for significance with repeated measures ANOVA. Survival percentages were arcsine-square root transformed. Pairwise comparisons of means were done by Student's $t$-tests.

\section{Results}

QS-independent killing of A. castellanii

in $\mathrm{P}$. aeruginosa biofilms

In flow-cell experiments, we observed that the amoeba $A$. castellanii was rapidly killed when colonizing pre-grown biofilms of $P$. aeruginosa PAO1. After settling on $P$. aeruginosa biofilms, amoeba numbers declined within $48 \mathrm{~h}$ from $80.2 \pm 7.8$ to $30.6 \pm 4.1$ cells per $\mathrm{mm}^{2}$ (Figure 1a). Microscopic inspection revealed that the lysis of $A$. castellanii trophozoites coincided with amoebae being colonized by $P$. aeruginosa. Both colonization and lysis of amoebal cells were also observed in biofilms of the QS-deficient rhlR/lasR mutant (Figure 1a). Differences between the wild-type and the rhlR/lasR mutants were not significant $(P>0.1)$, indicating that the killing of amoebae was QS independent. 

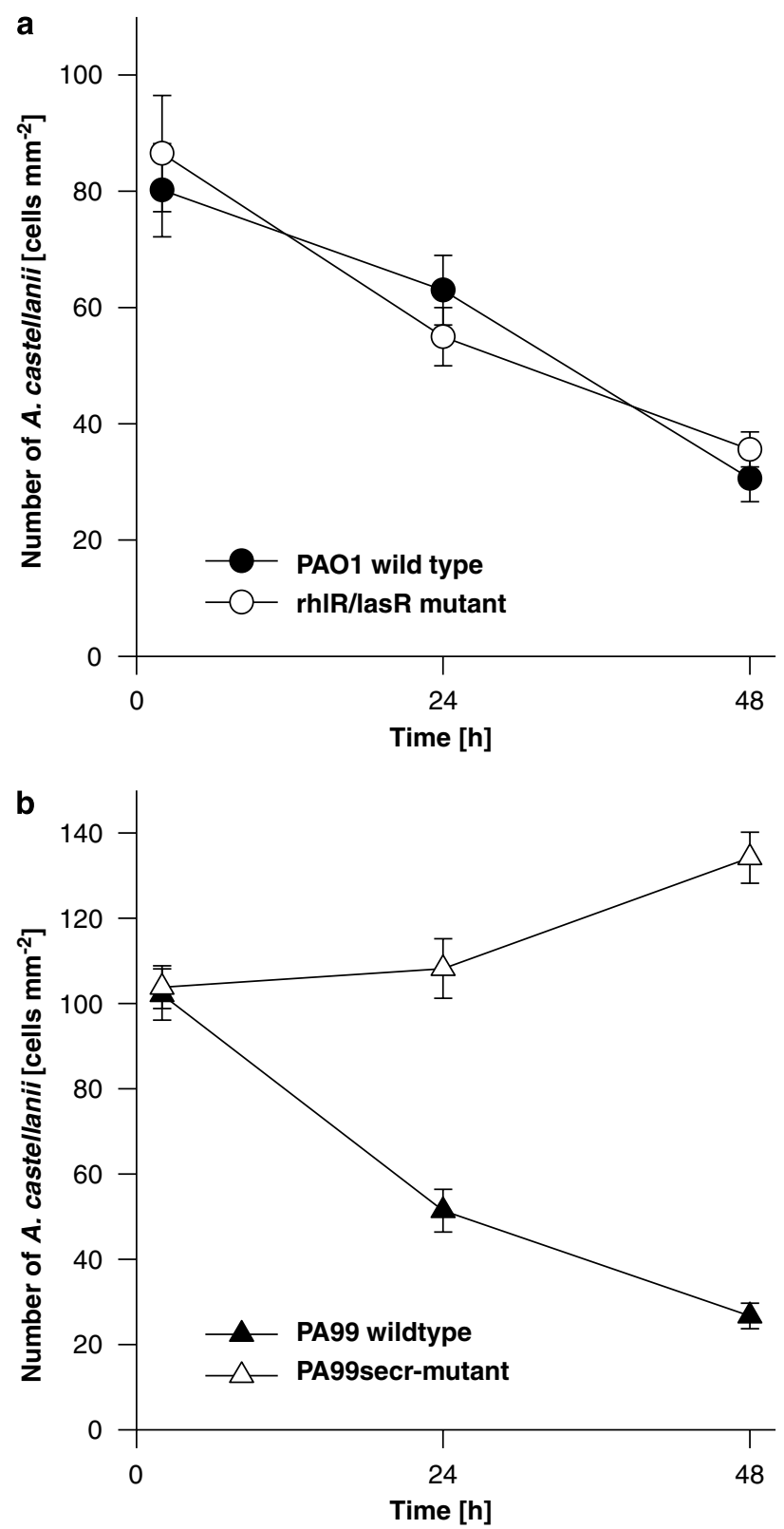

Figure 1 Lysis of Acanthamoeba castellanii settling on biofilms of Pseudomonas aeruginosa. Amoeba survival on (a) biofilms of $P$. aeruginosa PAO1 wild type versus QS-deficient rhlR/lasR mutant, and (b) biofilms of $P$. aeruginosa PA99 wild type versus T3SS-deficient PA99secr ${ }^{-}$mutant. Biofilms were pre-grown in flow-cell systems for 3 days prior to the addition of $A$. castellanii. Amoeba numbers include only structurally intact cells and are given as means \pm standard deviation $(n=3)$.

Amoeba-induced expression of T3SS-related genes To identify the underlying mechanism, we performed a transcriptomic analysis of $P$. aeruginosa PAO1 biofilms responding to $A$. castellanii cells. Analysis of the transcriptome profiles showed that the expression of the number of genes was significantly altered $(P<0.05)$ in response to $A$. castellanii when compared with the unexposed control biofilm. A total of 379 genes representing about $7 \%$ of the genome were differentially expressed $(\geqslant 3$-fold
Table 2 Expression of T3SS-related genes in Pseudomonas aeruginosa biofilms in response to the amoeba Acanthamoeba castellanii ( $\geqslant 3$-fold change in all three experiments)

\begin{tabular}{lllc}
\hline ID no. & Gene & $\begin{array}{l}\text { Product } \\
\text { function/ } \\
\text { description }\end{array}$ & Fold change \\
\hline PA1691 & $p s c T$ & $\begin{array}{l}\text { Translocation } \\
\text { protein in T3S }\end{array}$ & 4.3 \\
PA1692 & $p s c S$ & $\begin{array}{l}\text { Probable } \\
\text { translocation } \\
\text { protein in T3SS }\end{array}$ & 3.8 \\
PA1694 & $p s c Q$ & $\begin{array}{l}\text { Translocation } \\
\text { protein in T3S }\end{array}$ & 4.0 \\
PA1703 & $p c r D$ & $\begin{array}{l}\text { T3SS apparatus } \\
\text { protein }\end{array}$ & 3.8 \\
PA1707 & $p c r H$ & $\begin{array}{l}\text { Regulatory } \\
\text { protein }\end{array}$ & 4.7 \\
PA1710 & exsC & $\begin{array}{l}\text { ExsC } \\
\text { Transcriptional } \\
\text { regulator }\end{array}$ & 4.4 \\
PA1713 & exsA & $\begin{array}{l}\text { T3SS export } \\
\text { protein }\end{array}$ & 4.4 \\
PA1719 & $p s c F$ & & \\
& & &
\end{tabular}

change in all three experiments) with approximately $70 \%$ of genes being upregulated upon exposure to A. castellanii. Among the upregulated QS-independent genes with known function we found genes encoding proteins involved in the type III secretion, translocation and regulation machinery (Table 2): the genes pscTSQ of the operon PA1690-PA1697 (pscUTSRQPON), the gene psrD of the operon PA1698-PA1704 (popN-pcr1234DR), the gene pcrH of the operon PA1705-PA1709 (pcrGVH-popBD), the genes exsCA of the operon PA1710-PA1713 (exsCEBA) and the gene pscF of the operon PA1714-PA1725 (exsD-pscBCDEFGHIJKL). The amoeba-induced transcriptome did not indicate the involvement of QS-controlled virulence factors in the killing process (see Supplementary Information). Instead, two genes encoding enzymes with acyl-homoserine lactone acylase activity, quiP and pvdQ, were induced 16.4- and 4.6-fold, respectively, suggesting signal degradation rather than production. Exposure to amoebal attack also caused 3.9- to 16.6-fold enhanced expression levels in genes encoding nitric oxide reductases (norC and norB), which may contribute to the detoxification of amoeba-derived reactive nitrogen intermediates. Moreover, $P$. aeruginosa cells coexisting with amoebae in biofilms showed a 6.4-fold up-regulation of $f l i O$, a gene involved in flagellar biosynthesis and adherence to eukaryotic cells.

\section{Killing is cell contact-dependent and requires} a functional T3SS

To test for the involvement of T3SS components in the killing of biofilm-associated amoebae, we used previously characterized mutants of the clinical strain $P$. aeruginosa PA99, which secretes ExoS, 
ExoT and ExoU (Feltman et al., 2001; Shaver and Hauser, 2004; Table 1). In flow-cell experiments, we found that $A$. castellanii associated with biofilms of the $P$. aeruginosa PA99 wild type were lysed at a significantly higher rate than on biofilms of $P$. aeruginosa PAO1 (75.8 $\pm 5.1 \%$ decline within $48 \mathrm{~h}, P<0.01$, Figure 1b). In contrast, numbers of A. castellanii remained stable and even increased by about $30 \%$ when colonizing biofilms of the PA99secr ${ }^{-}$mutant, which does not produce any of the T3S proteins and is secretion apparatus defective.

On the basis of our observation that amoebae settling on $P$. aeruginosa biofilms become rapidly surrounded by highly motile planktonic bacteria before being colonized and eventually killed, we performed defined batch experiments, where $P$. aeruginosa suspensions were added to surfaceattached amoebae. Upon adding the $P$. aeruginosa PA99 wild type to $A$. castellanii in batch culture, we observed that highly motile cells of $P$. aeruginosa colonized the amoebae within $2-6 \mathrm{~h}$ to form dense biofilms surrounding the amoebal cells (Figure 2). After 24 h of incubation, about $97 \%$ of $A$. castellanii cells were colonized by the $P$. aeruginosa PA99 wild type (Figure 2a), which was followed by a substantial decline of amoebae numbers (12.7 $\pm 3.2 \%$ amoeba survival, Figure 2b). The PA99secr ${ }^{-}$strain, which does not produce any of the T3S proteins and is secretion apparatus defective, colonized $A$. castellanii cells to a similar extent (about $90 \%$ of the amoebae were colonized, $P=0.12$; Figure 2a) but did not cause a significant decrease in amoeba cell numbers (Figure 2b). Testing the effect of cell-free supernatant of $P$. aeruginosa, PA99 and PAO1 cultures revealed low cytotoxicity for both strains $(88.1 \pm 5.2 \%$ and $96.4 \pm 8.4 \%$ amoeba survival with PA99 and PAO1, respectively), which indicates that the killing of amoebae requires direct contact with bacterial cells.

T3SS-mediated cytotoxicity in $P$. aeruginosa is elicited by the combined action of secreted effector proteins, ExoS, ExoT, ExoU and ExoY. To determine the relative contribution of the type III effector proteins of $P$. aeruginosa to the killing of A. castellanii, isogenic mutants were screened, which secrete only one of the T3S effectors, ExoS, ExoT and ExoU (designated PA99S, PA99T and PA99U; Table 1). The ExoU-secreting strain PA99U caused significantly lower but still considerable mortality in A. castellanii populations (80.4 $\pm 3.2 \%$ compared to $87.3 \pm 3.1 \%$ in the wild type, $P=0.002$, Figure 3). Secretion of ExoS alone was also sufficient to kill $63.8 \pm 4.5 \%$ of the amoebae $(P<0.001)$. The double knock-out mutant PA99T, which secretes only ExoT, caused the greatest drop in virulence compared to the PA99 wild type resulting in $42.9 \pm 2.9 \%$ amoeba mortality $(P<0.001)$. On the basis of these data, we conclude that all three effector proteins contribute to the ability of $P$. aeruginosa to kill the amoeba $A$. castellanii.
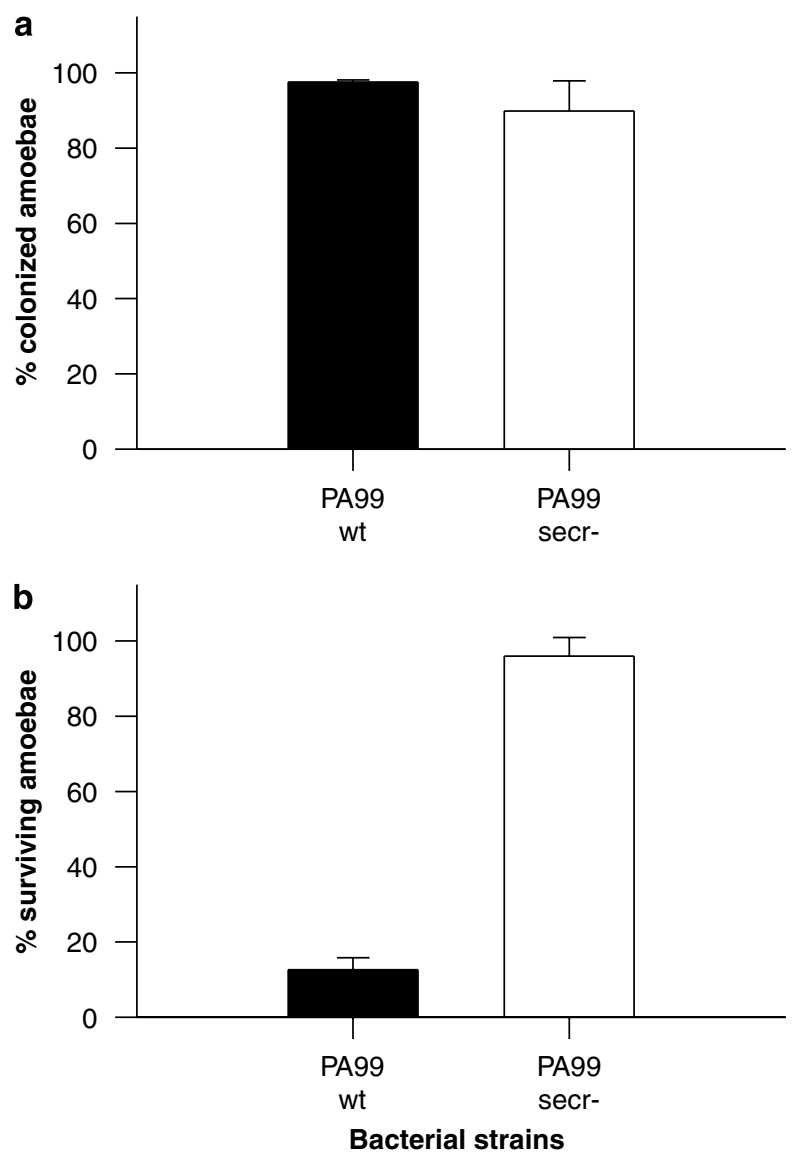

Figure 2 Colonization and killing of Acanthamoeba castellanii by Pseudomonas aeruginosa. (a) Colonization of $A$. castellanii by $P$. aeruginosa PA99 wild type and the T3SS-deficient mutant PA99secr ${ }^{-}$given as percentage of the total amoeba number after $24 \mathrm{~h}$. (b) Survival of A. castellanii after $24 \mathrm{~h}$ exposure to $P$. aeruginosa PA99 wild type and the T3SS-deficient mutant PA99 secr ${ }^{-}$given as percentage of the initial amoeba number. Error bars represent standard deviations $(n=5)$.

Regulation of T3SS-mediated cytotoxicity towards amoebae

Given the involvement of exopolymers, cell-to-cell communication and metabolic stresses in $P$. aeruginosa cytotoxicity and biofilm development, we examined regulator mutants defective in alginate biosynthesis, quorum sensing, adenosine $3^{\prime}, 5^{\prime}$-cyclic monophosphate (cAMP) signalling, and stationary phase sigma factors (Figure 4). As found in the flowcell studies, the QS-deficient rhlR/lasR mutant only showed a marginal difference to the PAO1 wild type in the batch experiments $(37.0 \pm 5.1 \%$ compared to $28.1 \pm 3.4 \%, P=0.04)$. The stationary phase sigma factor RpoS seems to be involved in the repression of the T3SS-mediated killing of $A$. castellanii as the rpoS mutant significantly increased amoebae mortality $(P=0.02)$. Modulation of intracellular cAMP levels in response to environmental signals is known to be another important mechanism controlling T3SS expression (Rietsch and Mekalanos, 2006; Wolfgang et al., 2003). Accordingly, we found that a 


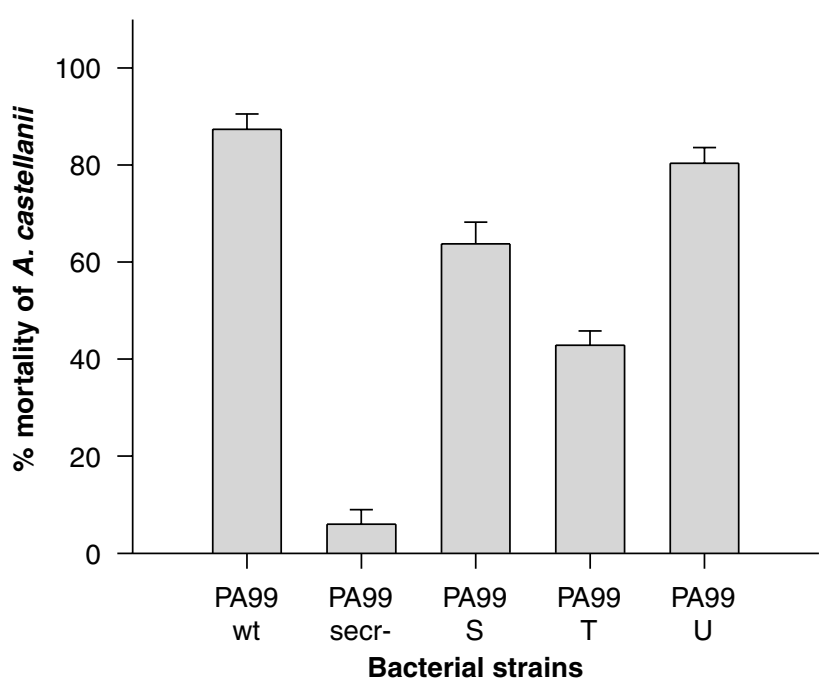

Figure 3 Contribution of type III secreted effectors to the killing of Acanthamoeba castellanii. Amoeba lysis is given as relative reduction of initial cell numbers after $24 \mathrm{~h}$ of co-incubation with Pseudomonas aeruginosa PA99 wild type and the T3SS-deficient mutant PA99secr- ${ }^{-}$, and mutants expressing only one of the three effectors, ExoS (PA99S), ExoT (PA99T) or ExoU (PA99U). Error bars represent standard deviations $(n=5)$.

mutant defective in the cAMP receptor protein Vfr caused substantially lower mortality in populations of $A$. castellanii $(63.1 \pm 6.8 \%, P<0.001)$. A complete loss of cytotoxicity was found in strain mucA22, lacking the functional anti-sigma factor MucA, which is involved in the repression of alginate biosynthesis. Strain mucA22 even caused a significant increase in amoebae numbers $(118.5 \pm 14.2 \%$, $P<0.001$ ), indicating the lack of toxicity and the feeding activity of $A$. castellanii on $P$. aeruginosa cells. Similarly, a mutant defective in the alternate sigma factor rpoN showed an increase in amoebae numbers $(120.8 \pm 7.8 \%, P<0.001)$, suggesting a central role of RpoN in the cytotoxicity towards amoebae.

\section{Discussion}

Our cocultivation experiments demonstrate that biofilms of $P$. aeruginosa respond to the colonization by environmental amoebae by upregulating genes of T3SS. Interestingly, recent studies have linked negative regulation of T3SS with factors thought to contribute to biofilm formation (Kuchma et al., 2005; Furukawa et al., 2006; Kulasakara et al., 2006; Laskowski and Kazmierczak, 2006; Ventre et al., 2006). This view is supported by our observation that amoebae settling on $P$. aeruginosa biofilms become rapidly surrounded by highly motile planktonic bacteria before being colonized and eventually killed (Figure 2). One global network affecting the reciprocal regulation of T3SS and biofilm formation is controlled by the RetS and LadS sensor proteins (Laskowski and Kazmierczak,

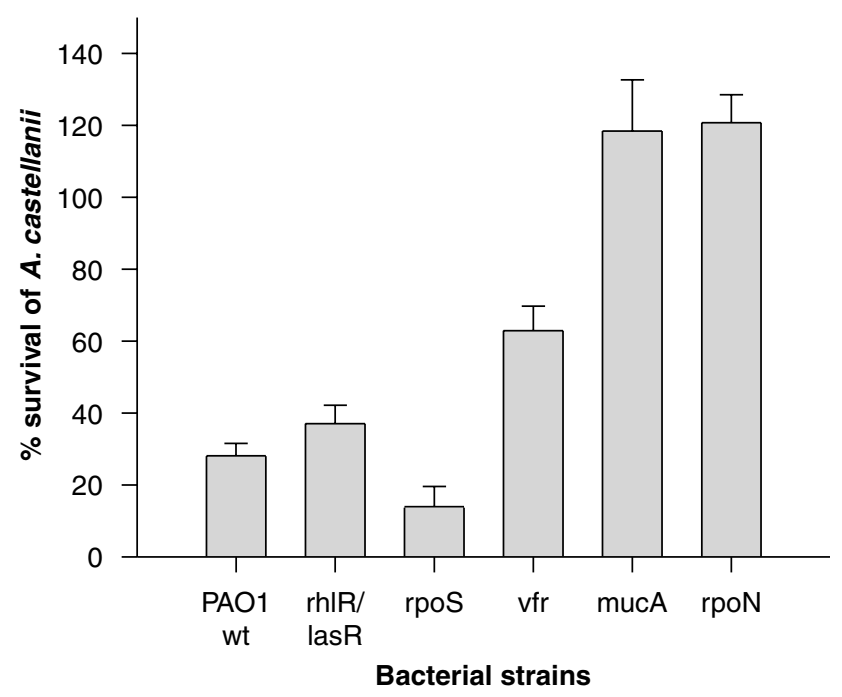

Figure 4 Contribution of global gene regulators to T3SSmediated killing of Acanthamoeba castellanii. Amoeba survival is given as relative change of initial cell numbers after $24 \mathrm{~h}$ of coincubation with Pseudomonas aeruginosa PAO1 wild type and mutants defective in rhlR/lasR, rpoS, vfr, mucA, rpoN. Error bars represent standard deviations $(n=5)$.

2006; Ventre et al., 2006). RetS is required for expression of the T3SS genes and for repression of exopolysaccharide biosynthesis, whereas LadS has the opposite effect. The exopolysaccharide alginate is further regulated by the alternative sigma factor AlgU, which is normally antagonized by the MucA anti-sigma factor. Mutation in the mucA gene is known to cause alginate overproduction, resulting in the mucoid phenotype. A recent study comparing global gene expression of mucA mutant versus wild type under T3SS-inducing conditions confirmed the downregulation of T3SS genes and upregulation of genes involved in alginate biosynthesis (Wu et al., 2004). In accordance with this, we found no decline in cell numbers of $A$. castellanii when cocultured with a mucA mutant (Figure 4), suggesting that matrix-encased cells do not colonize and kill the amoebae but a mobile subpopulation of bacteria which is transiently released from $P$. aeruginosa biofilms.

A recent study examining the contribution of the transcriptional anti-anti-activator ExsC to host contact-dependent T3SS gene expression found induction of the T3SS genes to be highly dependent on undefined host-cell factors (Dasgupta et al., 2006). Among the T3SS-related genes induced by $A$. castellanii we found a significant upregulation of ExsC (Table 2), which supports the notion that T3SS-mediated cytotoxicity to host cells other than mammalian cells is regulated through a generic ExsC-dependent pathway (Yahr and Wolfgang, 2006).

We have previously reported that biofilms of $P$. aeruginosa are protected against protozoan grazing through a QS-controlled inhibitory 
mechanism (Matz et al., 2004). However, indications for QS-independent response of $P$. aeruginosa were presented in an agar-plate based study on $P$. aeruginosa and the social amoeba Dictyostelium discoideum (Pukatzki et al., 2002). Besides the involvement of LasR in growth inhibition of the amoeba, the study described the lytic impact of the phospholipase ExoU on $D$. discoideum, suggesting the existence of two independent mechanisms acting in parallel. LasR is known to be without direct influence on the expression of T3SS genes (Dasgupta et al., 2003; Hogardt et al., 2004; Bleves et al., 2005). Mutants lacking the Rhl quorumsensing system, however, show increased expression of T3SS genes and secretion of ExoS at an earlier stage during exponential growth (Hogardt et al., 2004; Bleves et al., 2005). We found that a double knock out in the rhl/las system was without significant effect on cytotoxicity, suggesting a minor role of QS in this interaction. Notably, cocultivation of $P$. aeruginosa and amoebae in flow cells over relatively long time periods (10 days) leads to significant recovery in amoeba cell numbers associated with lasR/rhlR biofilms compared to wild-type biofilms (Matz et al., unpublished data). It appears that $P$. aeruginosa shows an instant response to the presence of amoeba by the active colonization and killing of amoebae and that QS-controlled mechanisms gain more significance during longer term coexistence at higher cell densities.

The differential significance of QS-dependent and independent mechanisms may find support in the emerging concept that T3SS is under strong transcriptional control of metabolic states (Dacheux et al., 2002; Wolfgang et al., 2003; Rietsch et al., 2004; Rietsch and Mekalanos, 2006). Growth conditions, cell densities and biofilm physiology may thus have a direct impact on which of the two pathways dominates. One transcriptional regulator that is induced at the transition from logarithmic to stationary growth phase is the sigma factor RpoS. About 770 genes are regulated by RpoS in the stationary phase of growth (Schuster et al., 2004). We found that an rpoS mutant had increased cytotoxicity against $A$. castellanii (Figure 4), which could be explained by the negative regulatory effect on T3SS expression reported for RpoS (Hogardt et al., 2004). Moreover, our transcriptome data reveal that $15 \%$ of the amoeba-induced genes are regulated by RpoS, among which are genes encoding T3SS components (ExsC, PcrH, PscF), two-component response regulators and chemotaxis transducer genes. Interestingly, algR transcription and alginate production have been shown to be RpoS-dependent (Suh et al., 1999; Schuster et al., 2004). As RpoS also regulates the Rhl system (Whiteley et al., 2000), transcriptional regulation by RpoS could be central to the timing of QS-independent and dependent mechanisms in the interaction of $P$. aeruginosa with biofilm-associated amoebae.
Modulation of intracellular cAMP levels in response to environmental signals is another important mechanism controlling T3SS expression. Studies using whole-genome microarray analyses revealed that mutants lacking cAMP or the cAMP receptor protein Vfr exhibited reduced expression of nearly 200 genes, including those involved in T3SS (Wolfgang et al., 2003). In our experiments, the vfr mutant was attenuated in killing $A$. castellanii (Figure 4), which supports the view that intracellular cAMP levels and the metabolic state of cells set the scene for $P$. aeruginosa to attack $A$. castellanii. Among the Vfr-regulated genes in the amoebainduced transcriptome ( $9 \%$ of total), we found genes encoding T3SS components (PcrH, PscF, PscQ, PscS, ExsA, ExsC) and the chemosensory signal transduction protein ChpA.

Interactions of $P$. aeruginosa with the fungus Candida albicans share some features such as the active colonization of filamentous cells and the formation of biofilms culminating in the lysis of C. albicans (Hogan and Kolter, 2002). The killing was reported to be multifactorial and under the control of the alternative sigma factor RpoN. Although T3SS components were not crucial to Pseudomonas-Candida interactions (R Kolter and D Hogan, personal communication), our data clearly indicate the central role of RpoN in the regulation of T3SS-mediated killing of $A$. castellanii (Figure 4). Our transcriptome data reveal that $12 \%$ of the amoeba-induced genes are regulated by RpoN (cf Dasgupta et al., 2003), but mostly encode hypothetical proteins with unknown function. Among the RpoN-regulated genes we find no evidence for their effect on flagella and pili biosynthesis. Interestingly, genes of the pel gene cluster, which is involved in polysaccharide biogenesis and early biofilm formation, show higher expression levels in an rpoN mutant (Dasgupta et al., 2003) and are found to be downregulated upon contact with $A$. castellanii. Assuming that higher biofilm-forming capacity is reciprocally linked to cytotoxicity, this could explain the higher survival and even growth of $A$. castellanii on biofilms of the rpoN mutant (Figure 4). P. aeruginosa mutants defective in rpoN are also significantly attenuated in other virulence models (Rahme et al., 1995; Tan et al., 1999; Hendrickson et al., 2001). However, although RpoN is known to be absolutely required for the expression of hrp genes encoding the components of a T3SS in Pseudomonas syringae (Hendrickson et al., 2000a, b), its role in the regulation of cytotoxicity is less clear in $P$. aeruginosa and awaits further elucidation.

To date, four type III effector proteins have been described to be injected by $P$. aeruginosa into the cytoplasm of eukaryotic cells: ExoU is a potent cytotoxin with phospholipase activity. ExoS and ExoT are bifunctional enzymes that have a small GTPase-activating protein domain and an ADPribosyltransferase domain, and inhibit phagocytosis 
by disrupting the actin cytoskeleton. ExoY is an adenylate cyclase causing elevated levels of cellular cAMP. Our experiments revealed that each one of ExoS, ExoT and ExoU contributes to amoebae killing (Figure 3). ExoU, however, appears to be the most toxic effector, which is confirmed by a study on $P$. aeruginosa virulence towards the social amoeba D. discoideum (Pukatzki et al., 2002), reporting loss of virulence in exoU and pscJ mutants and restored cytotoxicity in a complemented exoU mutant. Strains of $P$. aeruginosa have different combinations of genes encoding T3SS effectors. The lack of exoU in $P$. aeruginosa PAO1 (containing exoS, exoT and exoY) may explain the slightly lower cytotoxicity compared to that of strain PA99 (containing exoS, exoT and exoU; Figure 1).

The recent sequencing of many bacterial genomes has shown that T3SS are quite common among proteobacteria and Chlamydiae. Chlamydiae are notorious human pathogens with an obligate intracellular lifestyle but also include species living as endosymbionts of environmental amoebae (Wagner and Horn, 2006). Interestingly, genome analyses of the Acanthamoeba sp. endosymbiont Protochlamydia amoebophila UWE25 revealed that the common ancestor of pathogenic and symbiotic Chlamydiae has been intracellular for several hundreds of millions of years and contained a complete T3SS (Horn et al., 2004). Phylogenetic analysis of T3SS key components suggest that the T3SS evolved in the chlamydial lineage more than 700 million years ago in the interaction with early eukaryotes, which strengthens the notion that T3SS constitute an important module in past and present interactions between bacteria and protozoa.

As environmental pathogens like $P$. aeruginosa are integral members of natural microbial communities, it is anticipated that some virulence factors studied in the context of human disease may have their functional origin in the interaction with coexisting microbes. Toxins secreted by the P. aeruginosa T3SS have profound effects on the progression and severity of pneumonia in humans. Yet the pressures driving evolution of these toxins have been unclear, because humans are predominantly an accidental host of $P$. aeruginosa. Our findings suggest that the natural targets of this bacterium's type III secretion may be environmental protozoa. It seems that protozoa, particularly biofilm-associated amoeba, can induce exploitative behaviour in opportunistic bacterial pathogens and thus contribute to their pathogenic potential. Although it becomes obvious that multiple pathways exist in this interaction, future studies need to focus on the timing and regulatory fine tuning as well as the impact of environmental and metabolic conditions. Knowledge on the switch between persistence and aggression-or from a medical perspective between chronic and acute infection-is central to understand the opportunistic nature of pathogens like $P$. aeruginosa and, more generally, the functional role of natural biofilm communities as potential hotspots for eukaryote-targeting lifestyles.

\section{Acknowledgements}

This work was supported by grants from the German Research Foundation (to CM), the Danish Research Council for Technique and Production (to MG) and the German Mukoviszidose e.V. (to MG), grant R01 AI053674 from the National Institutes of Health (to ARH), the Otto Hahn fellowship of the Max Planck Society (to CM) and the Australian Cooperative Research Centre for Environmental Biotechnology and the Centre for Marine Bio-Innovation at the University of New South Wales (to SK).

\section{References}

Arndt H, Schmidt-Denter K, Auer B, Weitere M. (2003). Protozoans and biofilms. In: Krumbein WE, Paterson DM, Zavarzin GA (eds). Fossil and Recent Biofilms. Kluwer Academic Publishers: Dordrecht, pp 173-189.

Battin TJ, Kaplan LA, Denis Newbold J, Hansen CM. (2003). Contributions of microbial biofilms to ecosystem processes in stream mesocosms. Nature 426: 439-442.

Bleves S, Soscia C, Nogueira-Orlandi P, Lazdunski A, Filloux A. (2005). Quorum sensing negatively controls type III secretion regulon expression in Pseudomonas aeruginosa PAO1. J Bacteriol 187: 3898-3902.

Bodey GP, Bolivar R, Fainstein V, Jadeja L. (1993). Infections caused by Pseudomonas aeruginosa. Rev Infect Dis 5: 270-313.

Botzenhart K, Döring G. (1993). Ecology and epidemiology of Pseudomonas aeruginosa. In: Campa M, Bendinelli M, Friedman H (eds). Pseudomonas aeruginosa as an Opportunistic Pathogen. Plenum Press: New York, pp 1-18.

Costerton JW, Stewart PS, Greenberg EP. (1999). Bacterial biofilms: a common cause of persistent infections. Science 284: 1318-1322.

Dacheux D, Epaulard O, de Groot A, Guery B, Leberre R, Attree I et al. (2002). Activation of the Pseudomonas aeruginosa type III secretion system requires an intact pyruvate dehydrogenase $a c e A B$ operon. Infect Immun 70: 3973-3977.

Dasgupta N, Ashare A, Hunninghake GW, Yahr TL. (2006). Transcriptional induction of the Pseudomonas aeruginosa type III secretion system by low $\mathrm{Ca}^{2+}$ and host cell contact proceeds through two distinct signaling pathways. Infect Immun 74: 3334-3341.

Dasgupta N, Wolfgang MC, Goodman AL, Arora SK, Jyot J, Lory $\mathrm{S}$ et al. (2003). A four-tiered transcriptional regulatory circuit controls flagellar biogenesis in Pseudomonas aeruginosa. Mol Microbiol 50: 809-824.

Feltman H, Schulert G, Khan S, Jain M, Peterson L, Hauser AR. (2001). Prevalence of type III secretion genes in clinical and environmental isolates of Pseudomonas aeruginosa. Microbiology 147: 2659-2669.

Flanders JR, Yildiz FH. (2004). Biofilms as reservoirs for disease. In: Ghannoum M, O'Toole GA. (eds). Microbial Biofilms. ASM Press: Washington, DC, pp 314-331. 
Franklin M, Ohman D. (1993). Identification of algF in the alginate biosynthetic gene cluster of Pseudomonas aeruginosa which is required for alginate acetylation. J Bacteriol 175: 5057-5065.

Furukawa S, Kuchma SL, O’Toole GA. (2006). Keeping their options open: acute versus persistent infections. J Bacteriol 188: 1211-1217.

Hassell MP, Comins HN, May RM. (1994). Species coexistence and self-organizing spatial dynamics. Nature 370: 290-292.

He J, Baldini RL, Deziel E, Saucier M, Zhang Q, Liberati NT et al. (2004). The broad host range pathogen Pseudomonas aeruginosa strain PA14 carries two pathogenicity islands harboring plant and animal virulence genes. Proc Natl Acad Sci USA 101: 2530-2535.

Hendrickson EL, Guevera P, Ausubel FM. (2000a). The alternative sigma factor RpoN is required for hrp activity in Pseudomonas syringae pv. maculicola and acts at the level of hrpL transcription. J Bacteriol 182: 3508-3516.

Hendrickson EL, Guevera P, Penaloza-Vazquez A, Shao J, Bender C, Ausubel FM. (2000b). Virulence of the phytopathogen Pseudomonas syringae pv. maculicola is rpoN dependent. J Bacteriol 182: 3498-3507.

Hendrickson EL, Plotnikova J, Mahajan-Miklos S, Rahme LG, Ausubel FM. (2001). Differential roles of the Pseudomonas aeruginosa PA14 rpoN gene in pathogenicity in plants, nematodes, insects, and mice. J Bacteriol 183: 7126-7134.

Hogan DA, Kolter R. (2002). Pseudomonas-Candida interactions: an ecological role for virulence factors. Science 296: 2229-2232.

Hogardt M, Roeder M, Schreff AM, Eberl L, Heesemann J. (2004). Expression of Pseudomonas aeruginosa exoS is controlled by quorum sensing and RpoS. Microbiology 150: 843-851.

Horn M, Collingro A, Schmitz-Esser S, Beier CL, Purkhold U, Fartmann B et al. (2004). Illuminating the evolutionary history of Chlamydiae. Science 304: 728-730.

Jacobs MA, Alwood A, Thaipisuttikul I, Spencer D, Haugen E, Ernst S et al. (2003). Comprehensive transposon mutant library of Pseudomonas aeruginosa. Proc Natl Acad Sci USA 100: 14339-14344.

Kerr B, Riley MA, Feldman MW, Bohannan BJM. (2002). Local dispersal promotes biodiversity in a real-life game of rock-paper-scissors. Nature 418: 171-174.

Kimata N, Nishino T, Suzuki S, Kogure K. (2004). Pseudomonas aeruginosa isolated from marine environments in Tokyo Bay. Microb Ecol 47: 41-47.

Kuchma SL, Connolly JP, O'Toole GA. (2005). A threecomponent regulatory system regulates biofilm maturation and type III secretion in Pseudomonas aeruginosa. J Bacteriol 187: 1441-1454.

Kulasakara H, Lee V, Brencic A, Liberati N, Urbach J, Miyata S et al. (2006). Analysis of Pseudomonas aeruginosa diguanylate cyclases and phosphodiesterases reveals a role for bis- $\left(3^{\prime}-5^{\prime}\right)$-cyclic-GMP in virulence. Proc Natl Acad Sci USA 103: 2839-2844.

Laskowski MA, Kazmierczak BI. (2006). Mutational analysis of RetS, an unusual sensor kinase-response regulator hybrid required for Pseudomonas aeruginosa virulence. Infect Immun 74: 4462-4473.

Lau GW, Goumnerov BC, Walendziewicz CL, Hewitson J, Xiao W, Mahajan-Miklos S et al. (2003). The Drosophila melanogaster toll pathway participates in resistance to infection by the gram-negative human pathogen Pseudomonas aeruginosa. Infect Immun 71: 4059-4066.

Marciano-Cabral F, Cabral G. (2003). Acanthamoeba spp. as agents of disease in humans. Clin Microbiol Rev 16: 273-307.

Mathee K, Ciofu O, Sternberg C, Lindum PW, Campbell JIA, Jensen $\mathrm{P}$ et al. (1999). Mucoid conversion of Pseudomonas aeruginosa by hydrogen peroxide: a mechanism for virulence activation in the cystic fibrosis lung. Microbiology 145: 1349-1357.

Matz C, Bergfeld T, Rice SA, Kjelleberg S. (2004). Microcolonies, quorum sensing and cytotoxicity determine the survival of Pseudomonas aeruginosa biofilms exposed to protozoan grazing. Environ Microbiol 6: 218-226.

Matz C, Kjelleberg S. (2005). Off the hook-how bacteria survive protozoan grazing. Trends Microbiol 13: 302-307.

Matz C, McDougald D, Moreno AM, Yung PY, Yildiz FH, Kjelleberg S. (2005). Biofilm formation and phenotypic variation enhance predation-driven persistence of Vibrio cholerae. Proc Natl Acad Sci USA 102: 16819-16824.

Miyata S, Casey M, Frank DW, Ausubel FM, Drenkard E. (2003). Use of the Galleria mellonella caterpillar as a model host to study the role of the type III secretion system in Pseudomonas aeruginosa pathogenesis. Infect Immun 71: 2404-2413.

Molmeret M, Horn M, Wagner M, Santic M, Abu Kwaik Y. (2005). Amoebae as training grounds for intracellular bacterial pathogens. Appl Environ Microbiol 71: 20-28.

Parry JD. (2004). Protozoan grazing of freshwater biofilms. Adv Appl Microbiol 54: 167-196.

Pirnay JP, Matthijs S, Colak H, Chablain P, Bilocq F, Van Eldere J et al. (2005). Global Pseudomonas aeruginosa biodiversity as reflected in a Belgian river. Environ Microbiol 7: 969-980.

Plotnikova JM, Rahme LG, Ausubel FM. (2000). Pathogenesis of the human opportunistic pathogen Pseudomonas aeruginosa PA14 in Arabidopsis. Plant Physiol 124: 1766-1774.

Pukatzki S, Kessin RH, Mekalanos JJ. (2002). The human pathogen Pseudomonas aeruginosa utilizes conserved virulence pathways to infect the social amoeba Dictyostelium discoideum. Proc Natl Acad Sci USA 99: 3159-3164.

Rahme LG, Stevens EJ, Wolfort SF, Shao J, Tompkins RG, Ausubel FM. (1995). Common virulence factors for bacterial pathogenicity in plants and animals. Science 268: 1899-1902.

Rasmussen TB, Skindersoe ME, Bjarnsholt T, Phipps RK, Christensen KB, Jensen PO et al. (2005). Identity and effects of quorum-sensing inhibitors produced by Penicillium species. Microbiology 151: 1325-1340.

Rietsch A, Mekalanos JJ. (2006). Metabolic regulation of type III secretion gene expression in Pseudomonas aeruginosa. Mol Microbiol 59: 807-820.

Rietsch A, Wolfgang MC, Mekalanos JJ. (2004). Effect of metabolic imbalance on expression of type III secretion genes in Pseudomonas aeruginosa. Infect Immun 72: $1383-1390$.

Sambrook J, Fritsch EF, Maniatis T. (1989). Molecular Cloning: A Laboratory Manual. Cold Spring Harbor Laboratory Press: New York. 
Schuster M, Hawkins AC, Harwood CS, Greenberg EP. (2004). The Pseudomonas aeruginosa RpoS regulon and its relationship to quorum sensing. Mol Microbiol 51: 973-985.

Shaver CM, Hauser AR. (2004). Relative contributions of Pseudomonas aeruginosa ExoU, ExoS, and ExoT to virulence in the lung. Infect Immun 72: 6969-6977.

Sherr EB, Sherr BF. (2002). Significance of predation by protists in aquatic microbial food webs. Antonie Van Leeuwenhoek 81: 293-308.

Suh SJ, Silo-Suh L, Woods DE, Hassett DJ, West SEH, Ohman DE. (1999). Effect of rpoS mutation on the stress response and expression of virulence factors in Pseudomonas aeruginosa. J Bacteriol 181: 3890-3897.

Tan MW, Rahme LG, Sternberg JA, Tompkins RG, Ausubel FM. (1999). Pseudomonas aeruginosa killing of Caenorhabditis elegans used to identify $P$. aeruginosa virulence factors. Proc Natl Acad Sci USA 96: 2408-2413.

Thompson LS, Webb JS, Rice SA, Kjelleberg S. (2003). The alternative sigma factor RpoN regulates the quorum sensing gene rhlI in Pseudomonas aeruginosa. FEMS Microbiol Lett 220: 187-195.

Ventre I, Goodman AL, Vallet-Gely I, Vasseur P, Soscia C, Molin S et al. (2006). Multiple sensors control reciprocal expression of Pseudomonas aeruginosa regulatory RNA and virulence genes. Proc Natl Acad Sci USA 103: 171-176.

Wagner M, Horn M. (2006). The Planctomycetes, Verrucomicrobia, Chlamydiae and sister phyla comprise a superphylum with biotechnological and medical relevance. Curr Opin Biotechnol 17: 241-249.

Weitere M, Bergfeld T, Rice SA, Matz C, Kjelleberg S. (2005). Grazing resistance of Pseudomonas aeruginosa biofilms depends on type of protective mechanism, developmental stage and protozoan feeding mode. Environ Microbiol 7: 1593-1601.

Whiteley M, Parsek MR, Greenberg EP. (2000). Regulation of quorum sensing by RpoS in Pseudomonas aeruginosa. J Bacteriol 182: 4356-4360.

Wolfgang MC, Lee VT, Gilmore ME, Lory S. (2003). Coordinate regulation of bacterial virulence genes by a novel adenylate cyclase-dependent signaling pathway. Dev Cell 4: 253-263.

Wu W, Badrane H, Arora S, Baker HV, Jin S. (2004). MucAmediated coordination of type III secretion and alginate synthesis in Pseudomonas aeruginosa. $J$ Bacteriol 186: 7575-7585.

Yahr TL, Wolfgang MC. (2006). Transcriptional regulation of the Pseudomonas aeruginosa type III secretion system. Mol Microbiol 62: 631-640.

Supplementary Information accompanies the paper on The ISME Journal website (http://www.nature.com/ismej) 